\title{
Overall tolerability of Integrase Inhibitors in clinical practice: results from a multicenter Italian cohort
}

\author{
Arturo Ciccullo ${ }^{1,2}$, Gianmaria Baldin ${ }^{1,3 *}$, Vanni Borghi ${ }^{4}$, Gaetana Sterrantino ${ }^{5}$, Giordano \\ Madeddu ${ }^{6}$, Alessandra Latini ${ }^{7}$, Gabriella d' Ettorre $^{8}$, Alessandro Lanari ${ }^{9,10}$, Maria \\ Mazzitelli ${ }^{11}$, Manuela Colafigli ${ }^{7}$, Amedeo Capetti ${ }^{12}$, Letizia Oreni ${ }^{13}$, Filippo Lagi $^{5}$, Stefano \\ Rusconi $^{13}$, Simona Di Giambenedetto ${ }^{1,2}$
}

1 Section of Infectious Diseases, Department of Safety and Bioethics, Catholic University of the Sacred Heart, Rome, Italy

2 Fondazione Policlinico Universitario Agostino Gemelli IRCCS, Infectious Diseases Unit, Rome, Italy.

3 Mater Olbia Hospital, Olbia, Italy

4 Department of Infectious Diseases, Azienda Ospedaliero-Universitaria Policlinico of Modena, Modena, Italy;

5 Division of Tropical and Infectious Diseases, 'Careggi' Hospital, Florence, Italy;

6 Department of Clinical, Surgical and Experimental Sciences, University of Sassari, Sassari, Italy;

7 Infectious Dermatology and Allergology Unit, IFO S. Gallicano Institute (IRCCS), Rome, Italy;

8 Department of Public Health and Infectious Diseases, Azienda Policlinico Umberto I, Rome, Italy;

9 Infectious Diseases Unit, AOU Senese, Siena, Italy;

10 Department of Medical Biotechnologies, University of Siena, Siena, Italy;

11 Department of Health Sciences, Department of Medical and Surgical Sciences, Unit of Infectious and Tropical Diseases, Magna Graecia University, Catanzaro, Italy. 
12 Division of Infectious Diseases, Department of Infectious Diseases, Luigi Sacco University Hospital, Milan, Italy.

13 Infectious Diseases Unit, DIBIC Luigi Sacco, University of Milan, Milan, Italy;

${ }^{*}$ Corresponding author: Dr. Gianmaria Baldin, Istituto di Malattie Infettive, Università Cattolica del Sacro Cuore, Roma, Italy. Tel. 0039 06-30155366. E-mail: gian.baldin@gmail.com

Keywords: HIV, dolutegravir, raltegravir, elvitegravir, durability, integrase inhibitors Running Head: Overall tolerability of INIS 
Abstract

Background. International guidelines recommend the use of INI-based regimens as first line ARV in both naïve and experienced HIV-infected patients.

Materials and Methods. We analyzed a multicenter cohort of HIV-infected patients, both naïve and experienced, starting a ARV including an INI. Chi-square test and non-parametric tests were used to assess differences in categorical and continuous variables, respectively. Kaplan-Meyer survival analysis were performed to estimate the probability of maintaining the study-drug and Cox-regression analysis to evaluate predictors of discontinuation.

Results. We enrolled 4343 patients: 3143 (72.4\%) were males, with a median age of 49 years (IQR 41-55). Naïve patients were 733 (16.9\%), of whom 168 (22.9\%) AIDS-presenters. Overall, 2282 patients (52.5\%) started DTG, 1426 (32.8\%) RAL and 635 (14.7\%) EVG. During 10032 PYFU, we observed 1278 discontinuations (13 per 100PYFU); 448 of them (35\%) due to simplification and 355 (28\%) to toxicities (98 for CNS toxicity). Reasons of discontinuation were different between INIs. Estimated probability of maintaining DTG at 3 and 4 years were $81.5 \%(95 \% \mathrm{Cl} 80.5-82.5)$ and $76.3 \%(95 \% \mathrm{Cl} 73.9-78.7)$, respectively; RAL 61.6\% (95\%Cl 60.2-63.0) and 54.1\% (95\%Cl 52.7-55.5); EVG 71.6\% (95\%Cl 69.2-74.0) and 68.3\% (95\% Cl 65.3-71.3) ( $p<0.001)$. At a multivariable analysis, being on a RAL-based ARV (vs DTG, aHR 2.9, 95\%Cl 2.3-3.6, p<0.001), a EVG-based ARV (vs DTG, aHR 1.3 95\%Cl 1.1-1.7, $\mathrm{p}=0.049)$ and a peak HIV-RNA $>500 \mathrm{k} \mathrm{cp} / \mathrm{mL}(\mathrm{aHR} 1.3,95 \% \mathrm{Cl} 1.1-1.6, \mathrm{p}=0.006)$ predicted INI discontinuation.

Conclusions. Our data confirm the good tolerability of INIs in clinical practice. Differences emerge between the three drugs in reasons for discontinuation. 


\section{Introduction}

Integrase strand transfer Inhibitors (INI) have become, in both national and international guidelines [1,2], the first choice for both first line antiretroviral (ARV) regimens and switch regimen in virologically suppressed HIV-infected patients, thanks to their efficacy and favorable tolerability profile compared to other classes [3-5].

At the time of data collection, licensed INIs in Italy were raltegravir, elvitegravir/cobicistat and dolutegravir: raltegravir (RAL) was the first INI approved (US FDA, October 2007; EMA, January 2008) and as such was prevalently used in experienced, multi-failed patients, elvitegravir (EVG) was then approved, initially (FDA, August 2012; EMA, May 2013) exclusively as a component of a Single Tablet Regimen (STR), while dolutegravir (DTG), although being the most recently approved INI (FDA, August 2013; EMA, November 2013), has now become one of the most used antiretroviral drugs, thanks to its high genetic barrier and convenient dosing [6].

The efficacy of INI has been widely described in different works [7,8], but studies from clinical practice have questioned the optimal tolerability profile of this class of antiretroviral [9-11], showing unexpected high rates of toxicity-related discontinuations. However, data from large cohorts in particular regarding experienced patients are still lacking.

We aimed to compare, in a real-life scenario, the durability of all three INIs in both treatment-naive and experienced HIV-positive patients in our multicenter cohort (the ODOACRE Cohort) [12]. The Clinical centers involved in this study (listed in Table 1) take care of about 15.000 people living with HIV (PLWHIV), more than $10 \%$ of the estimated PLWHIV in Italy (130.000). [13] About half of them, at some point, started a INI-based strategy. In this study, we also intend to investigate reasons for INIs discontinuation and differences between them.

\section{Methods}

We retrospectively analyzed a cohort of HIV-1 infected adult (age $\geq 18$ years) patients, both treatment-naïve and experienced, from nine Italian clinical centers (the list of Clinical 
Centers is described in Table 1) belonging to the "ODOACRE cohort", starting an INI-based ARV regimen between January 2008 to December 2018. Primary endpoint was the time to treatment discontinuation (TD, defined as the discontinuation of either dolutegravir, raltegravir or elvitegravir, regardless of whether the remaining antiretroviral drugs used in the combination had been stopped or not).

We collected baseline characteristics (age, sex, HIV-risk factor), as well as patients' clinical history and viroimmunological parameters at baseline; reasons for INI discontinuations were also collected. Censor was defined as death, suspension of the INI or the date of the last virological determination.

Chi-square test and non-parametric tests were used to assess differences in categorical and continuous variables, as appropriate. Kaplan-Meyer survival analysis was performed to estimate the probability of maintaining the study-drug and Cox-regression analysis to evaluate predictors of discontinuation. Multi-variable models were adjusted for naïve status, calendar year and for significantly different variables between INIs at baseline.

The study was approved by each local Ethics Committee (protocol number of the promoting center: 5284/15) and all patients signed informed consent for data collection.

\section{Results}

We enrolled 4343 patients: 3143 (72.4\%) were males, with a median age of 49 years (Interquartile Range [IQR] 41-55). Median peak HIV-RNA was $5.04 \log _{10}$ copies/mL (IQR 4.50-5.51), while median CD4+ cell nadir was 171 cell/mm3 (IQR 48-304). Treatment-naïve patients were 733 (16.9\%), of whom 168 (22.9\%) were AIDS-presenters. As to experienced patients, their median time from HIV diagnosis was 16 years (IQR 8-23), with a median time from ARV initiation of 13 years (IQR 4-21); among experienced patients, 2696 (76.2\%) had a HIV-RNA $\leq 50 \mathrm{cp} / \mathrm{mL}$ at baseline. Overall, 2282 patients (52.5\%) started a DTGcontaining regimen, 1426 (33.1\%) a regimen containing RAL and 635 (14.7\%) EVG. Full patients' characteristics are available in Table 2.

During 10032 Patient Years of Follow-up (PYFU), we observed 1278 discontinuations, with a cumulative rate of 13 per 100 PYFU. Three hundred and thirty-one (14.5\%) discontinued 
DTG, 812 (56.9\%) discontinued RAL while 135 (21.3\%) discontinued EVG. Median time to INI discontinuation was 13 months (IQR 4-33). Discontinuations in the first year of followup ("early" discontinuations) were 572: 221 in the DTG group (66.7\% of group's overall discontinuations), 260 in the RAL group (32.0\%) and 91 in the EVG group (66.9\%).

Difference in discontinuation rate in the first year between the three analyzed groups was statistically significant $(p<0.001)$.

As to the reasons for INI discontinuations: 448 (35.0\% of total discontinuations) were due to simplification, 355 (27.8\%) to toxicities (98 of which were due to neuropsychiatric events), 145 (11.3\%) to virological failure, $69(5.4 \%)$ to death, $35(2.7 \%)$ to drug-drug interactions and $227(17.7 \%)$ to other/unknown causes. Reasons of discontinuation were different between INIs, as shown in Table 3.

Estimated probabilities of maintaining DTG at 2, 3 and 4 years were $84.7 \%(95 \% \mathrm{Cl}$ 83.985.5), $81.5 \%(95 \% \mathrm{Cl} 80.5-82.5)$ and $76.3 \%(95 \% \mathrm{Cl} 73.9-78.7)$, respectively; for RAL they were $69.2 \%(95 \% \mathrm{Cl} 67.9-70.5), 61.6 \%(95 \% \mathrm{Cl} 60.2-63.0)$ and $54.1 \%(95 \% \mathrm{Cl} 52.7-55.5)$, while for EVG were $77.2 \%(95 \% \mathrm{Cl} 75.8-78.6), 71.6 \%(95 \% \mathrm{Cl} 69.2-74.0)$ and $68.3 \%(95 \% \mathrm{Cl}$ 65.3-71.3) [Figure 1]. Differences between groups were statistically significant (Log-Rank $\mathrm{p}<0.001$ ). At a multivariable analysis, being on a RAL-based regimen (compared with DTG, aHR 2.9, 95\%Cl 2.3-3.6, p<0.001), a EVG-based one (vs DTG, aHR 1.3 95\%Cl 1.1-1.7, $\mathrm{p}=0.049$ ) and a peak HIV-RNA over 500.000 copies/mL (aHR 1.3, 95\%Cl 1.1-1.6, p=0.006) predicted INI discontinuation, after adjusting for calendar year, naïve status, age, sex, HIV risk factor, time of virological suppression, years of HIV and CD4+ cell nadir.

Considering only treatment-naive patients, probability of maintaining DTG was $78.7(95 \% \mathrm{Cl}$ $76.2-81.2)$ at 2 years, $77.1 \%(95 \% \mathrm{Cl} 74.4-79.8)$ at 3 years and $72.6 \%(95 \% \mathrm{Cl} 67.5-78.1)$ at 4 years. Probabilities for RAL were 54.1\% (95\% Cl 50.4-57.8), 45.6\% (95\% Cl 41.8-49.4) and $40.4 \%(95 \% \mathrm{Cl} 36.3-44.2)$, while for EVG they were $73.3 \%$ (95\% Cl 69.1-77.5), 65.6\% (95\% Cl 60.6-70.6) and 58.5\% (95\% $\mathrm{Cl}$ 52.0-65.0), respectively. In this sub-analysis, starting a firstline RAL-based regimen (compared with DTG, aHR 3.32, 95\% CI 2.1-5.2, p<0.001) and a concomitant AIDS-defining event (aHR $1.65,95 \% \mathrm{Cl} 1.01-2.71, \mathrm{p}=0.045)$ resulted predictors of INI discontinuation. 
Evaluating discontinuations due only to toxicities, we did not find a significant difference between INIs using Kaplan-Meyer survival analysis and Cox regression analysis (Log-Rank $\mathrm{p}=0.064$, Figure 2); in this specific analysis, male sex (vs female, aHR 0.65, 95\% $\mathrm{Cl} 0.44-0.96$, $p=0.030$ ) was reversely associated with drug discontinuation. Reasons for stopping study INI were significantly different between males and females $(p<0.001)$; in particular, among women, $8.7 \%$ of the total discontinuations were due to $\mathrm{Gl}$ toxicity, $2.3 \%$ to renal toxicity and $6.7 \%$ to neuropsychiatric events. Meanwhile, among males, the observed percentages were $5.4 \%, 3.5 \%$ and $8.2 \%$, respectively.

A specific sub-analysis on discontinuation due only to neuropsychiatric toxicity, found significant differences between groups (Log-rank $p<0.001$ ); a previous INI exposure (aHR $1.9,95 \% \mathrm{Cl} 1.1-3.3, \mathrm{p}=0.017)$ was associated with the event, while being on a RAL-based regimen (vs DTG, aHR 0.1, 95\% $\mathrm{Cl} 0.1-0.5, \mathrm{p}=0.004$ ) or on a EVG-based one (vs DTG, aHR $0.4,95 \% \mathrm{Cl} 0.2-0.9, \mathrm{p}=0.035$ ) was inversely associated.

We also observed significant differences between groups in estimated probabilities of INI discontinuations due to drug-drug interactions (Log-rank $\mathrm{p}<0.001$ ) and simplification (Logrank $\mathrm{p}<0.001$ ). In our cohort, being on a EVG-based regimen (vs DTG, aHR 8.4, 95\% $\mathrm{Cl} 3.0$ 23.0, $p<0.001$ ) independently predicted discontinuation due to drug-drug interaction. Discontinuation of study INI due to simplification, meanwhile, was predicted by being on a RAL-based regimen (vs DTG, aHR 20.0, 95\%Cl 11.3-35.2, p<0.001) or a EVG-based one (vs DTG, aHR 2.4, 95\% Cl 1.1-5.7, p=0.042) while it was inversely associated with time of ARV exposure (aHR 0.96, 95\% $\mathrm{Cl} 0.92-0.98, \mathrm{p}=0.024)$. As to patients discontinuing RAL due to simplification, 258 of them (65.0\%) started a ARV regimen containing another INI (177 with DTG).

\section{Discussion}

In our multicenter cohort we found differences in durability of the three integrase inhibitors available in Italy at the time of data analysis. Overall, the probability of maintaining DTG was higher compared with those of RAL and EVG both in treatment experienced and naïve patients, a finding in line with previous studies [14,15]. However, no difference was found regarding discontinuations due to toxicity between groups, 
confirming the good tolerability of the whole integrase inhibitors drug class observed in other cohort studies [16].

Central nervous system toxicity was a particular concern, not only for patients taking dolutegravir [17], but also for those on other INIs [11]. In our cohort, we found that patients taking DTG-based regimens were more prone to discontinue following neuropsychiatric events compared with the other INIs, a finding that confirms results from other cohorts $[18,19]$. CNS toxicity was the leading cause of DTG interruption in our cohort.

However, as recently pointed out by Llibre et al. [20], there is the possibility that Clinicians' awareness, following initial reports on the association between DTG and neuropsychiatric events, had a significant biasing effect. On the other hand, the favorable dosing and the high efficacy shown as part of 2DR [21,22], make DTG the drug less likely to be stopped due to simplification.

The higher probability of stopping EVG due to drug-drug interaction could be explained by the need of a pharmacokinetic booster.

Early ( $\leq 1$ year) discontinuations were lower in the RAL group compared with the other two. This finding could be explained by the different use that was made of raltegravir when it was first approved; being the first INI available, in fact, led clinicians in using RAL prevalently in heavily experienced patients, while DTG and EVG in our cohort were started mainly because of simplification or dyslipidemia.

Strengths of our study include the size of the population, one of the largest described, representing different clinical experiences, as well as the length of follow-up; these features make it possible to best reflect clinical practice. As to the main limitations, the most important is the high number of discontinuations due to unknown reasons. In addition, the three groups are very different, mainly due to the fact that the three molecules were introduced into the clinical practice in different time points and were used often in the context of different ARV strategies, although during analysis we tried to correct this bias, by adjusting for differences between groups. Finally, other limitations are 
the retrospective design of the study and the fact that adverse events not leading to drug discontinuations were not collected.

In conclusion, our data confirm the good tolerability of INIs in clinical practice, with some differences between the three analyzed drugs regarding reasons for discontinuation.

\section{Acknowledgment}

Collaborators in ODOACRE group: Alberto Borghetti, Roberto Cauda, Luigi Celani, Alex Dusina, Silvia Lamonica, Francesca Lombardi, Claudio Maria Mastroianni, Davide Moschese, Enrica Tamburrini (Roma), Arianna Emiliozzi, Barbara Rossetti (Siena), Cristina Mussini (Modena), Sergio Babudieri, Andrea De Vito (Sassari), Carlo Torti, Enrico Maria Trecarichi (Catanzaro), Maria Vittoria Cossu, Massimo Galli, Andrea Giacomelli, Giuliano Rizzardini (Milano)

\section{Author Contribution statement}

$\mathrm{ACi}, \mathrm{GB}$ and SDG contributed to the conception and design of the study. ACi and GB contributed to the draft of the paper. VB, GS, GM, ALat, GdE, Alan, MM, MC, Aca, LO, FL and SR contributed to the acquisition of the data. ACi, GB and SDG contributed to the analysis and interpretation of data. GS, GM, SR and SDG contributed to the critical revision of the paper for important intellectual content. All Authors approved the final version of the paper.

\section{Transparency declaration}

GB received travel grant from Gilead Sciences. GS has received funds for speaking by Gilead, Merk, Janssen, Abbvie, ViiV. GM reports personal fees from Gilead Sciences, Janssen-Cilag,Merck Sharp \& Dohme, ViiV Healthcare, outside the submitted work. ACa has received a personal grant from $A B$, Gilead and ViiV. SR received research grants to his Institution from ViiV Heathcare, Gilead Sciences and Janssen, outside the submitted work; he was also a paid consultant for ViiV Heathcare, Gilead Sciences, Merck Sharp and Dohme, Bristol-Myers Squibb and Janssen. SDG was a paid consultant or member of advisory boards for Gilead, ViiV Healthcare, Janssen-Cilag, Merck Sharp \& Dohme and 
Bristol-Myers Squibb. All other authors ( $A C i, V B, G d E, A L, M M, M C, L O, F L)$ : none to declare.

\section{Funding}

This study was conducted during our routine clinical activity 


\section{References}

1) Italian guidelines for the use of antiretroviral agents and the diagnostic-clinical management of HIV-1 infected persons. Edition 2017. http://www.salute.gov.it/imgs/C 17 pubblicazioni 2696 allegato.pdf

2) EACS Guidelines 9.1 - October 2018. http://www.eacsociety.org/files/2018 guidelines-9.1-english.pdf

3) Blanco JL, Whitlock G, Milinkovic A et al. HIV integrase inhibitors: a new era in the treatment of HIV. Expert Opin Pharmacother. 2015 Jun;16(9):1313-24.

4) Walmsley S, Baumgarten A, Berenguer J et al. Brief Report: Dolutegravir Plus Abacavir/Lamivudine for the Treatment of HIV-1 Infection in Antiretroviral TherapyNaive Patients: Week 96 and Week 144 Results From the SINGLE Randomized Clinical Trial. J Acquir Immune Defic Syndr. 2015 Dec 15;70(5):515-9.

5) Baldin G, Ciccullo A, Capetti A et al. Efficacy and safety of switching to dolutegravir plus emtricitabine/tenofovir disoproxil fumarate (TDF) or elvitegravir/cobicistat/emtricitabine/TDF in virologically suppressed HIV-infected patients in clinical practice: results from a multicentre, observational study. HIV Med. 2019 Feb;20(2):164-168.

6) Kandel CE, Walmsley SL. Dolutegravir - a review of the pharmacology, efficacy, and safety in the treatment of HIV. Drug Des Devel Ther. 2015 Jul 7;9:3547-55. doi: 10.2147/DDDT.S84850. eCollection 2015. Review.

7) Kanters S, Vitoria $M$, Doherty $M$ et al. Comparative efficacy and safety of first-line antiretroviral therapy for the treatment of HIV infection: a systematic review and network meta-analysis. Lancet HIV. 2016 Nov;3(11):e510-e520. doi: 10.1016/S2352-3018(16)30091-1.

8) Psichogiou M, Poulakou G, Basoulis D et al. Recent Advances in Antiretroviral Agents: Potent Integrase Inhibitors. Curr Pharm Des. 2017;23(18):2552-2567.

9) De Boer $M$, van den Berk GE, van Holten $N$ et al. Intolerance of dolutegravir containing cART regimens in real life clinical practice. AIDS. 2016;30:2831-4. 
10) Squillace N, Ricci E, Quirino T et al. (2017) Safety and tolerability of elvitegravir/Cobicistat/Emtricitabine/Tenofovir Disoproxil fumarate in a real life setting: Data from surveillance cohort long-term toxicity antiretrovirals/antivirals (SCOLTA) project. PLoS ONE 12(6): e0179254

11) Madeddu G, Menzaghi B, Ricci E et al. Raltegravir central nervous system tolerability in clinical practice: results from a multicenter observational study. AIDS. 2012 Nov 28;26(18):2412-5.

12) Ciccullo A, Baldin G, Capetti A, et al. Cohort profile: The Observational cohort for the study of DOlutegravir in Antiretroviral Combination REgimens (ODOACRE). $\underline{B M J}$ Open. 2019;9(12):e029960. Published 2019 Dec 2. doi:10.1136/bmjopen-2019029960

13) Laura Camoni, Vincenza Regine, Karen Stanecki, Maria Cristina Salfa, Mariangela Raimondo, Barbara Suligoi, "Estimates of the Number of People Living with HIV in Italy", BioMed Research International, vol. 2014, Article ID 209619, 6 pages, 2014. https://doi.org/10.1155/2014/209619

14) d'Arminio Monforte A, Cozzi-Lepri A, Di Biagio A et al. Durability of first-line regimens including integrase strand transfer inhibitors (INSTIS): data from a real-life setting. J Antimicrob Chemother. 2019 Jan 29.

15) Rusconi S, Adorni F, Tau P et al. Dolutegravir (DTG)-containing regimens after receiving raltegravir (RAL) or elvitegravir (EVG): Durability and virological response in a large Italian HIV drug resistance network (ARCA). J Clin Virol. 2018 Aug;105:112-117.

16) Peñafiel J, de Lazzari $E$, Padilla $M$, et al. Tolerability of integrase inhibitors in a reallife setting. Journal of Antimicrobial Chemotherapy, 72(6), 1752-1759. doi:10.1093/jac/dkx053

17) Hoffmann C, Welz T, Sabranski M et al. Higher rates of neuropsychiatric adverse events leading to dolutegravir discontinuation in women and older patients. HIV Med. 2017;18(1):56-63. 
18) Cid-Silva $P$, Llibre JM, Fernández-Bargiela N, Margusino-Framiñán L et al. Clinical Experience with the Integrase Inhibitors Dolutegravir and Elvitegravir in HIVinfected Patients: Efficacy, Safety and Tolerance. Basic Clin Pharmacol Toxicol. 2017 Nov;121(5):442-446.

19) Elzi L, Erb S, Furrer H, Cavassini $M$ et al. Adverse events of raltegravir and dolutegravir. AIDS. 2017 Aug 24;31(13):1853-1858.

20) Llibre JM, Montoliu A, Miró JM et al. Discontinuation of dolutegravir, elvitegravir/cobicistat and raltegravir because of toxicity in a prospective cohort. HIV Med. 2019 Mar;20(3):237-247.

21) Baldin G, Ciccullo A, Borghetti A et al. Virological efficacy of dual therapy with lamivudine and dolutegravir in HIV-1-infected virologically suppressed patients: long-term data from clinical practice. J Antimicrob Chemother. 2019 Feb 5.

22) Boswell R, Foisy MM, Hughes CA. Dolutegravir Dual Therapy as Maintenance Treatment in HIV-Infected Patients: A Review. Ann Pharmacother. 2018 Jul;52(7):681-689.

23) Italian guidelines for the use of antiretroviral agents and the diagnostic-clinical management of HIV-1 infected persons. Edition 2017. http://www.salute.gov.it/imgs/C 17 pubblicazioni_2696_allegato.pdf

24) EACS Guidelines 9.1 - October 2018. http://www.eacsociety.org/files/2018 guidelines-9.1-english.pdf

25) Blanco JL, Whitlock G, Milinkovic A et al. HIV integrase inhibitors: a new era in the treatment of HIV. Expert Opin Pharmacother. 2015 Jun;16(9):1313-24.

26) Walmsley S, Baumgarten A, Berenguer J et al. Brief Report: Dolutegravir Plus Abacavir/Lamivudine for the Treatment of HIV-1 Infection in Antiretroviral TherapyNaive Patients: Week 96 and Week 144 Results From the SINGLE Randomized Clinical Trial. J Acquir Immune Defic Syndr. 2015 Dec 15;70(5):515-9.

27) Baldin G, Ciccullo A, Capetti A et al. Efficacy and safety of switching to dolutegravir plus emtricitabine/tenofovir disoproxil fumarate (TDF) or elvitegravir/cobicistat/emtricitabine/TDF in virologically suppressed HIV-infected patients in clinical practice: results from a multicentre, observational study. $\underline{\text { HIV }}$ Med. 2019 Feb;20(2):164-168. 
28) Kandel CE, Walmsley SL. Dolutegravir - a review of the pharmacology, efficacy, and safety in the treatment of HIV. Drug Des Devel Ther. 2015 Jul 7;9:3547-55. doi: 10.2147/DDDT.S84850. eCollection 2015. Review.

29) Kanters S, Vitoria $M$, Doherty $M$ et al. Comparative efficacy and safety of first-line antiretroviral therapy for the treatment of HIV infection: a systematic review and network meta-analysis. Lancet HIV. 2016 Nov;3(11):e510-e520. doi: 10.1016/S2352-3018(16)30091-1.

30) Psichogiou M, Poulakou G, Basoulis D et al. Recent Advances in Antiretroviral Agents: Potent Integrase Inhibitors. Curr Pharm Des. 2017;23(18):2552-2567.

31) De Boer $M$, van den Berk GE, van Holten $N$ et al. Intolerance of dolutegravir containing cART regimens in real life clinical practice. AIDS. 2016;30:2831-4.

32) Squillace N, Ricci E, Quirino T et al. (2017) Safety and tolerability of elvitegravir/Cobicistat/Emtricitabine/Tenofovir Disoproxil fumarate in a real life setting: Data from surveillance cohort long-term toxicity antiretrovirals/antivirals (SCOLTA) project. PLoS ONE 12(6): e0179254

33) Madeddu G, Menzaghi B, Ricci E et al. Raltegravir central nervous system tolerability in clinical practice: results from a multicenter observational study. AIDS. 2012 Nov 28;26(18):2412-5.

34) Ciccullo A, Baldin G, Capetti A, et al. Cohort profile: The Observational cohort for the study of DOlutegravir in Antiretroviral Combination REgimens (ODOACRE). $\underline{B M J}$ Open. 2019;9(12):e029960. Published 2019 Dec 2. doi:10.1136/bmjopen-2019029960

35) Laura Camoni, Vincenza Regine, Karen Stanecki, Maria Cristina Salfa, Mariangela Raimondo, Barbara Suligoi, "Estimates of the Number of People Living with HIV in Italy", BioMed Research International, vol. 2014, Article ID 209619, 6 pages, 2014. https://doi.org/10.1155/2014/209619

36) d'Arminio Monforte A, Cozzi-Lepri A, Di Biagio A et al. Durability of first-line regimens including integrase strand transfer inhibitors (INSTIS): data from a real-life setting. J Antimicrob Chemother. 2019 Jan 29. 
37) Rusconi S, Adorni F, Tau P et al. Dolutegravir (DTG)-containing regimens after receiving raltegravir (RAL) or elvitegravir (EVG): Durability and virological response in a large Italian HIV drug resistance network (ARCA). J Clin Virol. 2018 Aug;105:112-117.

38) Peñafiel J, de Lazzari E, Padilla M, et al. Tolerability of integrase inhibitors in a reallife setting. Journal of Antimicrobial Chemotherapy, 72(6), 1752-1759. doi:10.1093/jac/dkx053

39) Hoffmann C, Welz T, Sabranski M et al. Higher rates of neuropsychiatric adverse events leading to dolutegravir discontinuation in women and older patients. $\underline{\text { HIV }}$ Med. 2017;18(1):56-63.

40) Cid-Silva $P$, Llibre JM, Fernández-Bargiela N, Margusino-Framiñán L et al. Clinical Experience with the Integrase Inhibitors Dolutegravir and Elvitegravir in HIVinfected Patients: Efficacy, Safety and Tolerance. Basic Clin Pharmacol Toxicol. 2017 Nov;121(5):442-446.

41) Elzi L, Erb S, Furrer $H$, Cavassini $M$ et al. Adverse events of raltegravir and dolutegravir. AIDS. 2017 Aug 24;31(13):1853-1858.

42) Llibre JM, Montoliu A, Miró JM et al. Discontinuation of dolutegravir, elvitegravir/cobicistat and raltegravir because of toxicity in a prospective cohort. HIV Med. 2019 Mar;20(3):237-247.

43) Baldin G, Ciccullo A, Borghetti A et al. Virological efficacy of dual therapy with lamivudine and dolutegravir in HIV-1-infected virologically suppressed patients: long-term data from clinical practice. J Antimicrob Chemother. 2019 Feb 5.

44) Boswell R, Foisy MM, Hughes CA. Dolutegravir Dual Therapy as Maintenance Treatment in HIV-Infected Patients: A Review. Ann Pharmacother. 2018 Jul;52(7):681-689. 
Table 1. List of Clinical Centers involved in the study

Catholic University of the Sacred Heart, Fondazione Policlinico Universitario Agostino Gemelli IRCCS, Rome

Azienda Ospedaliero Universitaria di Modena, Clinica Malattie Infettive e Tropicali, Modena

Division of Tropical and Infectious Diseases, 'Careggi' Hospital, Florence

Department of Clinical, Surgical and Experimental Sciences, University of Sassari, Sassari

Infectious Dermatology and Allergology Unit, IFO S. Gallicano Institute (IRCCS), Rome

Department of Public Health and Infectious Diseases, Azienda Policlinico Umberto I, Rome

Infectious Diseases Unit, AOU Senese, Siena

Department of Health Sciences, Department of Medical and Surgical Sciences, Unit of Infectious and Tropical Diseases, Magna Graecia University, Catanzaro

Division of Infectious Diseases, Department of Infectious Diseases, Luigi Sacco University Hospital, Milan 
Table 2. Characteristics of the study population.

\begin{tabular}{|c|c|c|c|c|c|}
\hline Variables & $\begin{array}{l}\text { Overall } \\
n=4343\end{array}$ & $\begin{array}{l}\text { Dolutegravir } \\
\qquad \mathrm{n}=2282\end{array}$ & $\begin{array}{l}\text { Raltegravir } \\
\text { n=1426 }\end{array}$ & $\begin{array}{l}\text { Elvitegravir } \\
\qquad \mathrm{n}=635\end{array}$ & $\mathrm{p}$ \\
\hline Males, n (\%) & $\begin{array}{c}3143 \\
(72.4 \%)\end{array}$ & $1698(74.4)$ & $961(67.4)$ & $484(76.2)$ & $<0.001$ \\
\hline $\begin{array}{l}\text { Age, Years, Median } \\
\text { (IQR) }\end{array}$ & $\begin{array}{c}48.9(41.2- \\
54.8)\end{array}$ & $\begin{array}{c}50.0(41.3- \\
55.7)\end{array}$ & $\begin{array}{c}48.0(42.3- \\
53.7)\end{array}$ & $\begin{array}{c}47.2(36.8- \\
54.2)\end{array}$ & $<0.001$ \\
\hline $\begin{array}{l}\text { Risk factors, n (\%) } \\
\text { Heterosexual } \\
\text { MSM } \\
\text { IDU } \\
\text { Others/Unknown }\end{array}$ & $\begin{array}{l}1684(38.8) \\
1540(35.5) \\
771(17.8) \\
348(8.0)\end{array}$ & $\begin{array}{l}898(39.4) \\
856(37.5) \\
314(13.8) \\
214(9.4)\end{array}$ & $\begin{array}{l}555(38.9) \\
395(27.7) \\
387(27.1) \\
89(6.2)\end{array}$ & $\begin{array}{l}231(36.4) \\
289(45.5) \\
70(11.0) \\
45(7.1)\end{array}$ & $<0.001$ \\
\hline HCV Ab positive, n (\%) & 733 (18.9) & $295(14.3)$ & $378(30.6)$ & $60(10.5)$ & $<0.001$ \\
\hline HBsAg positive, n (\%) & $122(3.2)$ & $50(2.5)$ & $42(3.4)$ & $30(5.4)$ & 0.002 \\
\hline CDC stage $\mathrm{C}, \mathrm{n}(\%)$ & $1244(28.9)$ & $619(27.6)$ & $462(32.4)$ & $163(25.7)$ & 0.001 \\
\hline $\begin{array}{l}\text { Years from HIV } \\
\text { diagnosis, Median } \\
\text { (IQR) }\end{array}$ & $\begin{array}{c}13.5(3.8- \\
21.4)\end{array}$ & $\begin{array}{c}13.2(3.9- \\
21.6)\end{array}$ & $\begin{array}{c}15.1(5.5- \\
21.9)\end{array}$ & $\begin{array}{c}9.9(1.5- \\
19.6)\end{array}$ & $<0.001$ \\
\hline $\begin{array}{l}\text { Zenith HIV-RNA, } \log _{10} \\
\mathrm{cp} / \mathrm{ml} \text {, Median (IQR) }\end{array}$ & $\begin{array}{c}5.04(4.50- \\
5.51)\end{array}$ & $\begin{array}{c}5.05(4.49- \\
5.51)\end{array}$ & $\begin{array}{c}5.03(4.51- \\
5.51)\end{array}$ & $\begin{array}{c}5.07(4.52- \\
5.52)\end{array}$ & 0.894 \\
\hline $\begin{array}{l}\text { Nadir CD4+, } \\
\text { cells/mmc, Median } \\
\text { (IQR) }\end{array}$ & $\begin{array}{c}171(48- \\
304)\end{array}$ & $181(48-316)$ & $\begin{array}{c}154(46- \\
272)\end{array}$ & $\begin{array}{c}189(58- \\
327)\end{array}$ & $<0.001$ \\
\hline $\begin{array}{l}\text { Baseline CD4+, } \\
\text { cells/mmc, Median } \\
\text { (IQR) }\end{array}$ & $\begin{array}{c}550(329- \\
788)\end{array}$ & $\begin{array}{c}610(396- \\
840)\end{array}$ & $\begin{array}{c}469(270- \\
699)\end{array}$ & $\begin{array}{c}520(320- \\
752)\end{array}$ & $<0.001$ \\
\hline $\begin{array}{l}\text { Reasons for starting } \\
\text { study drug, } n(\%) \\
\text { Naive } \\
\text { Treatment failure }\end{array}$ & $\begin{array}{l}734(16.9) \\
515(11.9)\end{array}$ & $\begin{array}{l}378(16.6) \\
214(9.4)\end{array}$ & $\begin{array}{l}213(14.9) \\
257(18.0)\end{array}$ & $\begin{array}{c}143(22.5) \\
44(6.9)\end{array}$ & $<0.001$ \\
\hline
\end{tabular}


18

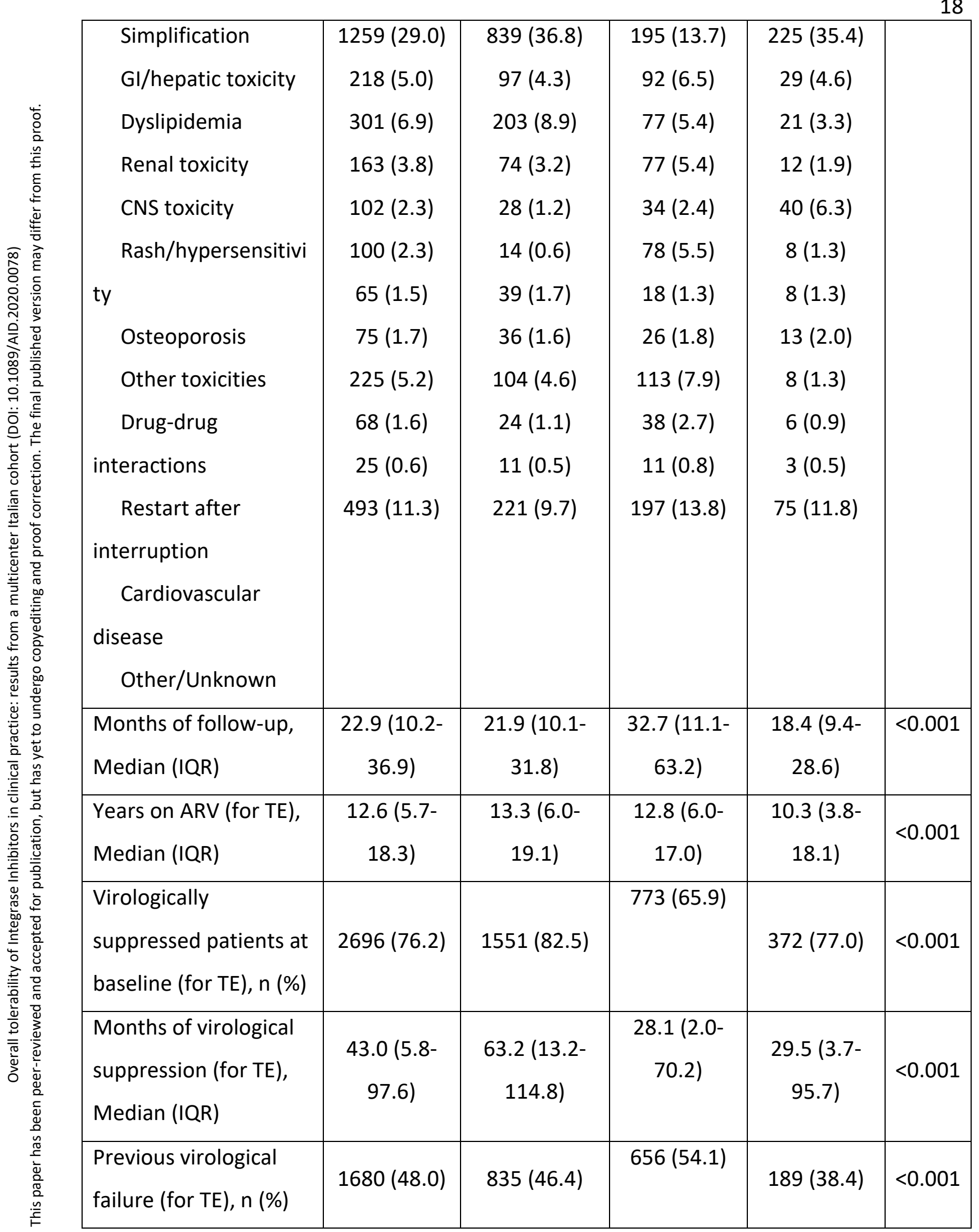


19

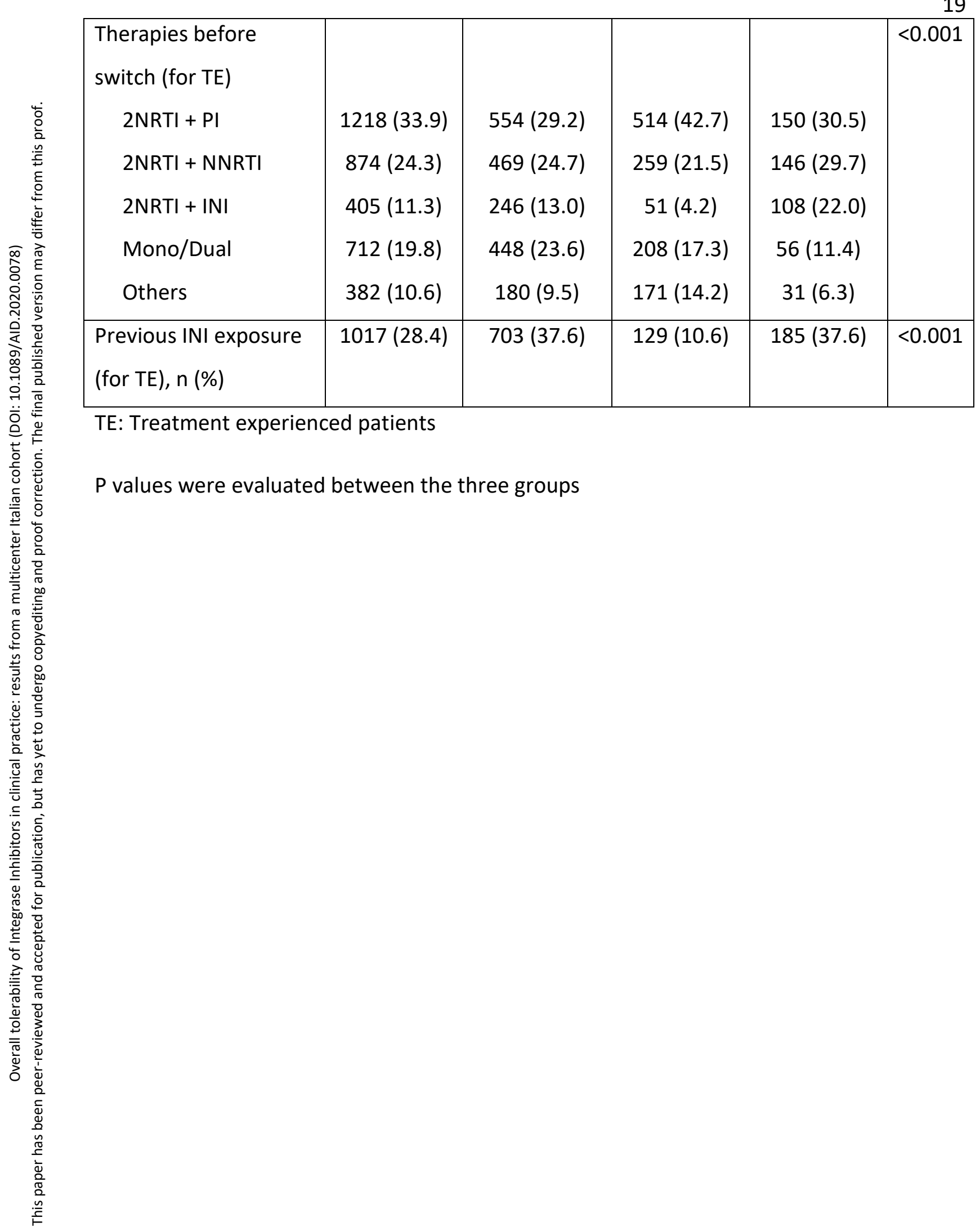


Table 3. Reasons for INI discontinuation

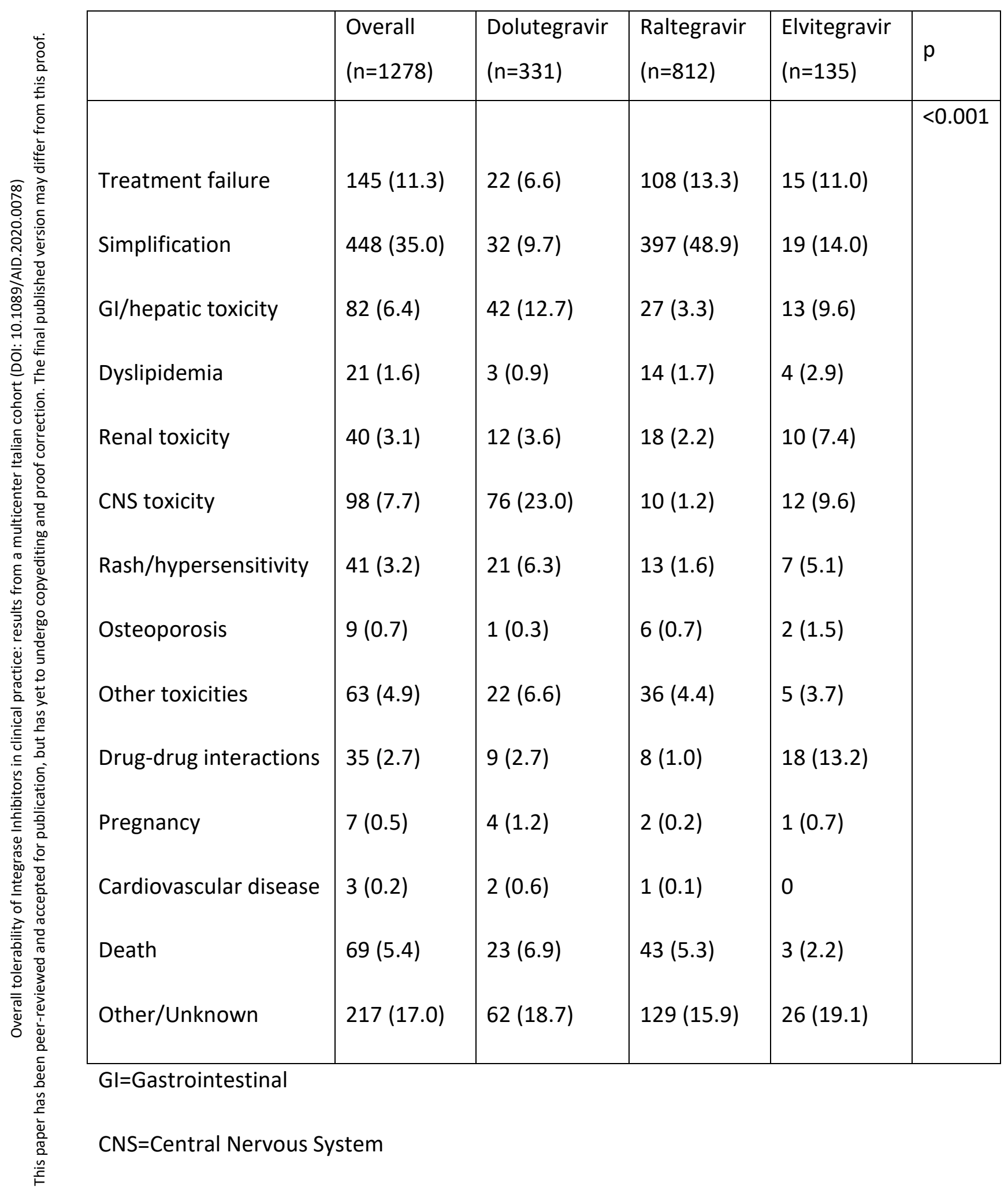


Figure Legends

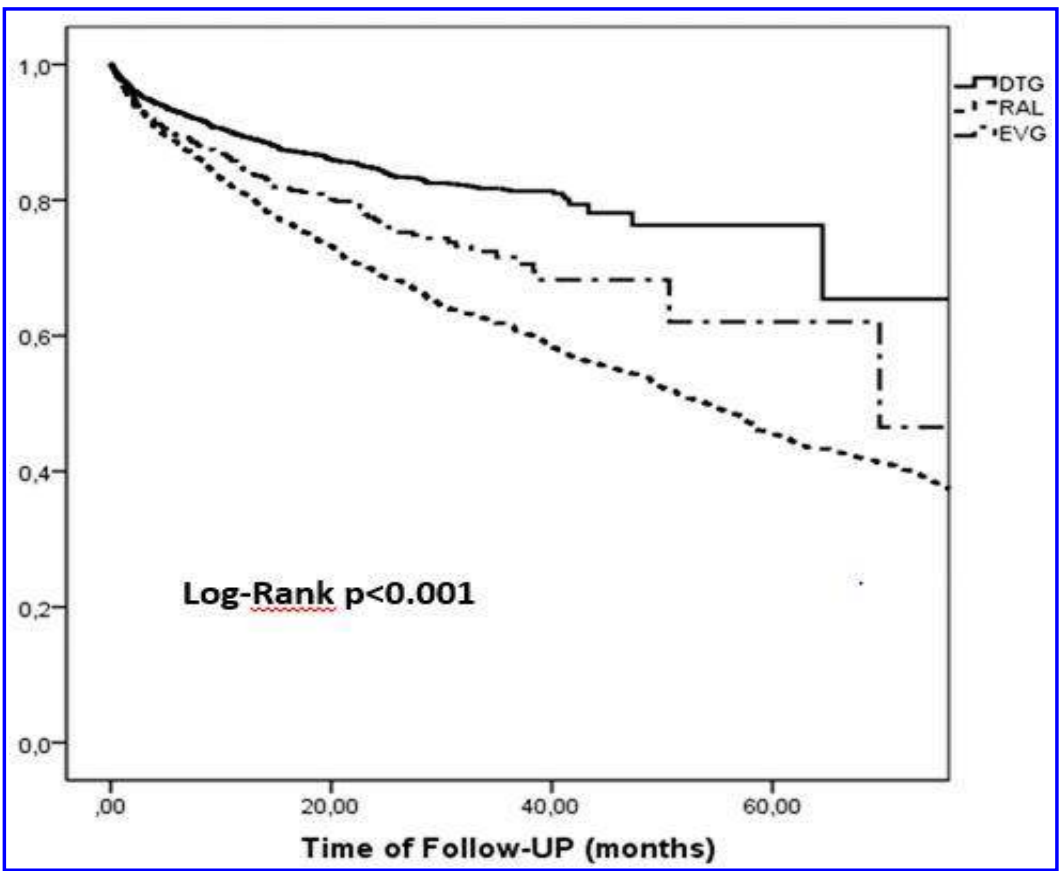

Figure 1. Estimated probability of maintaining study drug (Overall population) 


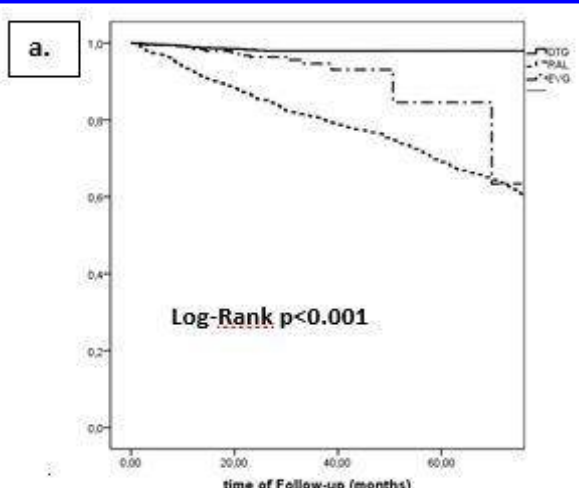

b.
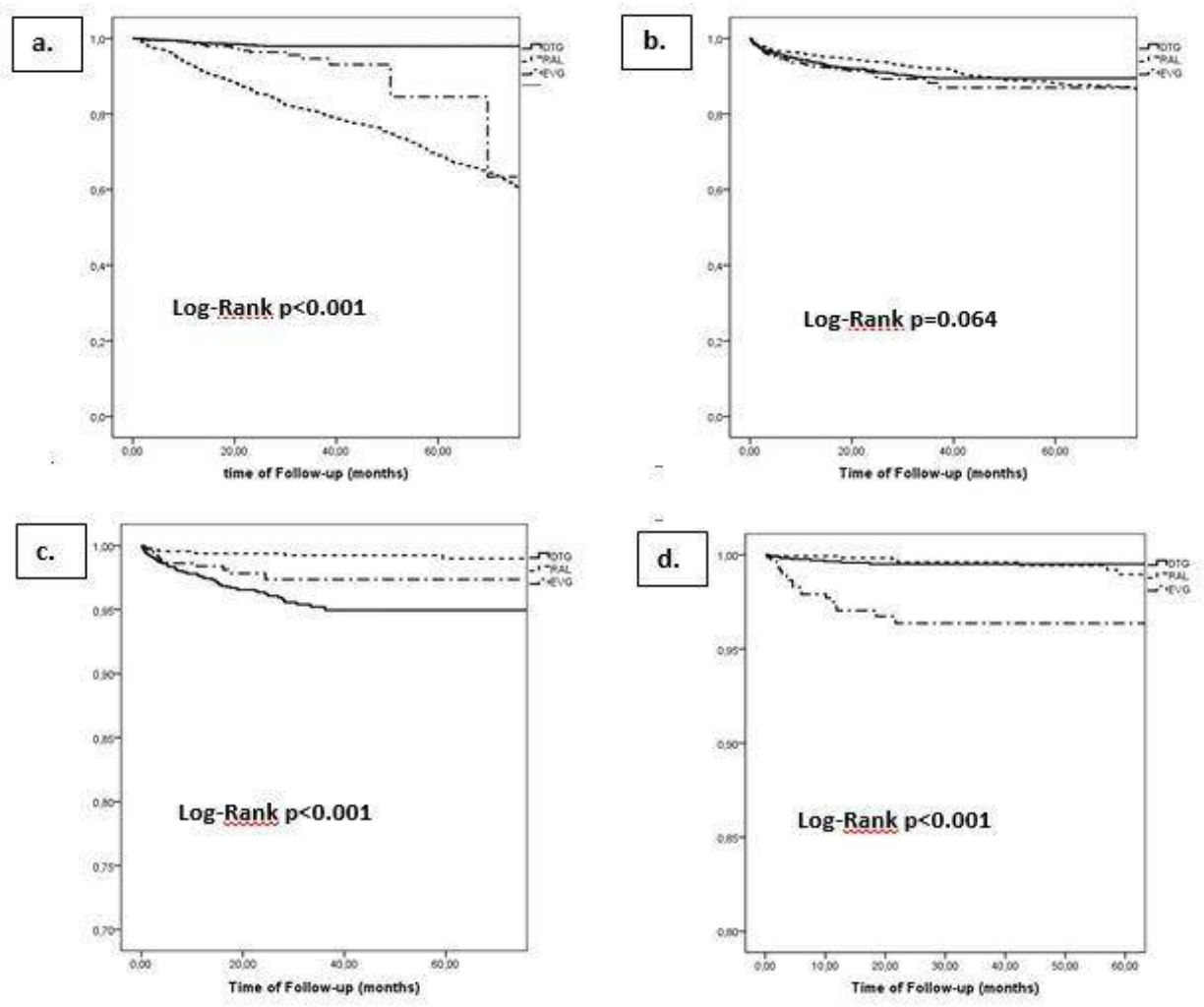

Figure 2. Estimated probabilities of maintatining study drugs (Sub-analysis): a.

discontinuations due to treatment simplification. b. discontinuations due to overall toxicity. c. discontinutions due to neuropsychiatric events. d. discontinuations due to drugdrug interactions. 